\title{
Laparoscopic vaginal vault closure with conventional straight instruments in single-port access total laparoscopic hysterectomy
}

\author{
Daehyun Park', Juyoung Kim², Hye Sun Jun², Hyangjin Jeong ${ }^{3}$, Youngse Park ${ }^{2}$ \\ ${ }^{1}$ Department of Obstetrics and Gynecology, Bundang CHA Hospital, Seongnam; ${ }^{2}$ Department of Obstetrics and Gynecology, Gangnam CHA Hospital, \\ CHA University, Seoul; ${ }^{3}$ Department of Nursing, Saekyung College, Yeongwol, Korea
}

\section{Objective}

Laparoscopic vaginal vault closure with conventional straight instruments is the final barrier to single-port access total laparoscopic hysterectomy (SPA-TLH). The aim of this study is to find out the safer, easier, simpler, faster, and even cheaper way to overcome it.

\section{Methods}

Vaginal vault suturing techniques of 152 consecutive single-port access total laparoscopic hysterectomy cases performed by the author in Gangnam CHA Hospital, CHA University from October 1, 2003 to June 30, 2012, were retrospectively analysed with medical records and DVDs.

\section{Results}

Of 152 patients who were attempted SPA-TLH, 119 patients (78\%) were finished their operations without conversion to multi-port laparoscopy or laparotomy. Of women with successful SPA-TLH, 8 cases $(7 \%)$ were closed their vaginal vaults vaginally (median, 20 minutes; range, 15-44 minutes), and 111 cases (93\%) laparoscopically (median, 44 minutes; range, 13-56 minutes). Laparoscopic vault closure techniques were continuous suture (4 cases, 3\%; median, 36 minutes; range, 30-45 minutes), interrupted sutures using knot-pusher (7 cases, 6\%; median, 52 minutes; range, 48-56 minutes) Endo Stitch suture (2 cases, 2\%; median, 32 minutes; range, 13-50 minutes), continuous vault closure using percutaneous sling sutures (PCSS) (92 cases, 77\%; median, 40 minutes; range, 19-56 minutes), and continuous vault closure without PCSS (6 cases, 5\%; median, 23 minutes; range, 16-31 minutes).

\section{Conclusion}

Laparoscopic vault closure using PCSS in SPA-TLH only with conventional straight instruments is the best way to overcome the barrier and the short-cut to shorten the learning curve to date.

Keywords: Hysterectomy; Laparoscopy; Suture technique

\section{Introduction}

Reich et al. [1] first performed laparoscopic assisted vaginal hysterectomy (LAVH) in 1989 to patients that were unable to undergo total vaginal hysterectomy, and successfully conducted total laparoscopic hysterectomy (TLH) in 1994. Singleport access total laparoscopic hysterectomy (SPA-TLH) was first performed in 2008 and has rapidly replaced conventional laparoscopic surgery [2].

Along with the introduction of SPA-TLH, current multi-port
Received: 2012.11.9. Revised: 2013.5.9. Accepted: 2013.5.16.

Corresponding author: Youngse Park

Department of Obstetrics and Gynecology, Gangnam CHA

Hospital, CHA University, 566 Nonhyeon-ro, Gangnam-gu, Seoul 135-081, Korea

Tel: +82-2-3468-2804 Fax: +82-2-3468-2638

E-mail: yspark0108@empas.com

Articles published in Obstet Gynecol Sci are open-access, distributed under the terms of the Creative Commons Attribution Non-Commercial License (http://creativecommons. org/licenses/by-nc/3.0/) which permits unrestricted non-commercial use, distribution, and reproduction in any medium, provided the original work is properly cited.

Copyright ( 2013 Korean Society of Obstetrics and Gynecology 


\title{
Obstetrics \& Gynecology Science
}

\author{
Vol. 56, No. 6, 2013
}
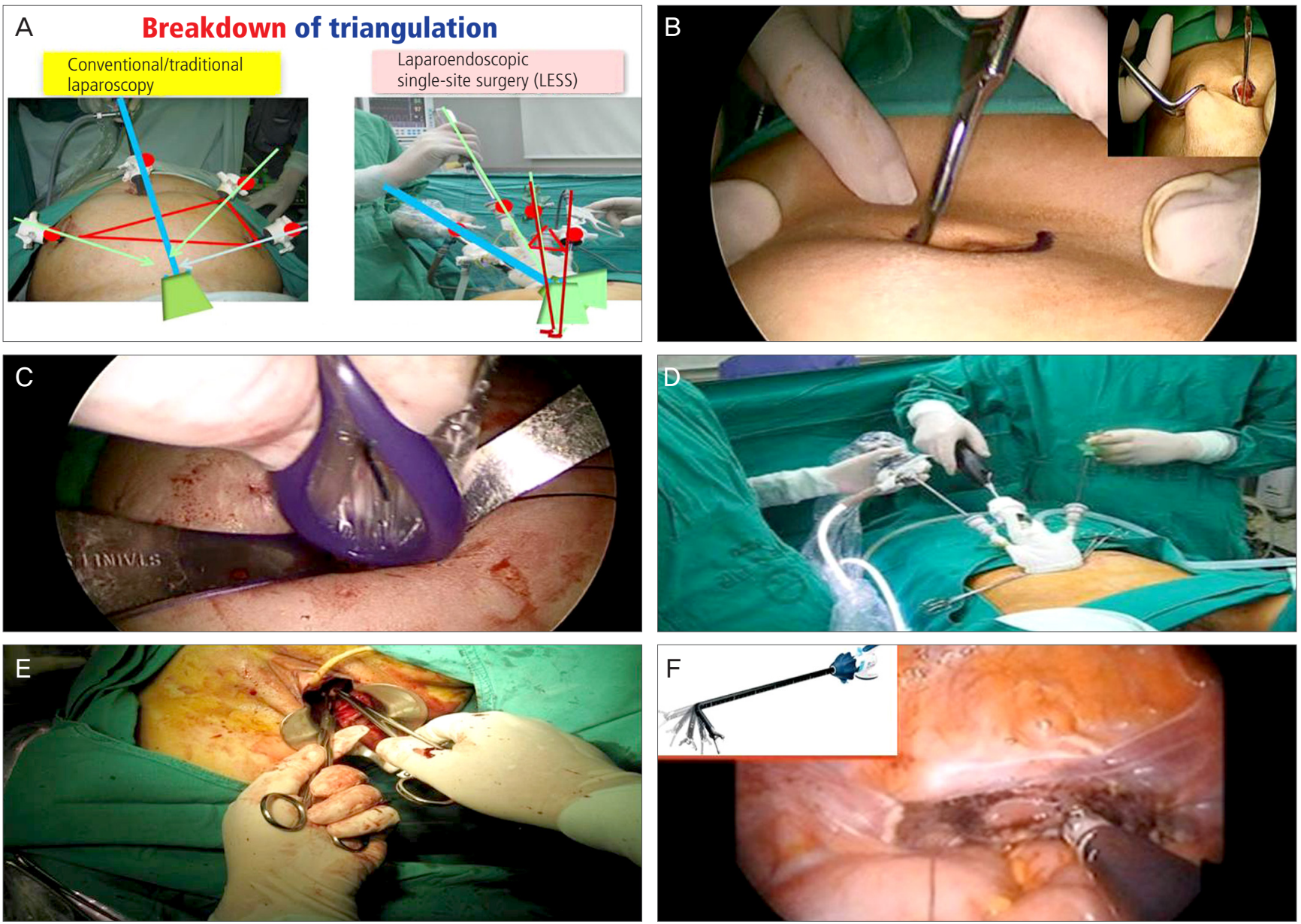

Fig. 1. (A) Breakdown of triangulation in single port access surgery. (B) Semi-lunar incision (vertical incision: left upper). (C) Insertion of Allexis retraction ring. (D) Finial view of home-made port. (E) Vaginal repair. (F) Endo Stitch suture (articulating ES: left).

laparoscopic surgery which uses 3 to 4 ports are now called conventional or traditional laparoscopy. Single-port access laparoscopic surgery performed by making a single skin incision in umbilical region is called scarless surgery along with natural orifice translumenal endoscopic surgery due to the surgical scar located on the umbilicus which is almost unseen $[3,4]$.

The fundamental notions of a single-port laparoscopic surgery are all laparoscopic working ports are located in the same incision [5]. For this reason, the single-port access surgery has several limitations including breakdown of triangulation, in-line view, crowding of surgical instruments, crossing/ sword-fighting instruments, and others compared to multiport surgery (Fig. 1A) [3,4].

Pelosi and Pelosi [6] first performed single-port LAVH and bilateral salpingo-oophorectomy in 1991. This surgery was not commonly performed due to its complex surgical procedure.
The definition of TLH has been changing. Hysterectomy consists of 7 steps regardless of their routes [7] including 1) transection of bilateral round ligaments and dissection of broad ligaments, 2) transection of utero-ovarian ligaments for preverving ovaries, or transection of infundibulo-pelvic ligaments for sacrificing ovaries, 3) transecton of uterine vessels, 4) bladder dissection, 5) transection of both cardinal-uterosacral ligament complexes, 6) vaginal wall incision (colpotomy) and amputation of uterus, 7) vaginal vault closure.

Therefore, the procedures of TLH are modified into 9 steps, which are as follows [8].

1) Insertion of uterine manipulator, 2) installation of ports and exploration of abdominal/pelvic cavity, 3) transection of ovarian ligaments/infundibulo-pelvic ligaments and round ligaments, 4) transection of cardinal ligaments ( \pm dissection of ureter/uterine vessels), 5) bladder dissection, 6) vaginal wall incision (colpotomy) (+uterosacral ligament transection), 7) 


\section{Obstetrics \& Gynecology Science}

Daehyun Park, et al. Laparoscopic vaginal vault closure in SPA-TLH

morcellation of uterus, 8) retrieval of uterus, 9) suture of vaginal vault and pelvic peritoneum.

In the early stage of TLH, all steps of TLH were done laparoscopically, except for 7) morcellation of uterus, 8) retrieval of uterus, and 9) vault closure. However, Reich, the founder of LAVH and TLH proposed that the vault closure and colposuspension needs to be done laparoscopically to prevent postoperative vaginal vault prolapse in TLH in the 38th American Association of Gynecologic Laparoscopy held in November, 2009. He also suggested that 7) morcellation of the uterus and 8 ) retrieval of the uterus could be performed through the vagina [3].

The most difficult procedure of laparoscopic surgery is suturing. Suturing in a single-port laparoscopic surgery is more complex than that in multi-port laparoscopic surgery. Laparoscopic vaginal vault suture is the final barrier to SPA-TLH [7].

The aim of this study is to find out the safer, easier, simpler, faster, and even cheaper way to overcome it only with conventional straight instruments.

\section{Materials and methods}

\section{Subjects and methods}

Retrospective analysis of medical records and DVDs of 152 consecutive cases of SPA-TLH was performed by the author in Gangnam CHA Hospital, CHA University from October 2009 to June 2012.

The inclusion criterion of performing SPA-TLH aligns with 4-port TLH. The study excluded patients 1) those unable to maintain Trendelenburg position for a long time due to severe cardiovascular diseases, 2) those unable to take dorsal lithotomy position due to pelvic abnormality, 3) history of abdominal surgery or severe abdominal adhesions due to pelvic inflammation (three or more occurrences of Caesarean section), 4) severe endometriosis, 5) large myoma uteri/adenomyosis, 6) malignant tumor, and 7) obese female (body mass index >25) from undergoing TLH. In the initial stage of single-port surgery, the size of uterus was limited to a minimum of twelve gestational weeks, but gradually increased to larger uterus.

Of 152 patients who attempted SPA-TLH, 119 patients (78\%) finished their surgeries successfully (24 cases of multiport conversion, 9 cases of laparotomy conversion). The authors analyzed the amount of time, problems, complications, and other factors according to vault closure techniques per- formed in successful SPA-TLH.

Vault closure time was measured from the point of insertion of the suture material equipped with needle holder into the abdominal cavity to the cutting the knot with the scissors after the final ligation. In case of vaginal suture, time taken to change the position of the surgeon was included.

\section{Surgical method}

Uterine manipulator (RUMI, Cooper Surgical, Trumbull, CT, USA) was inserted, and then a $15 \mathrm{~mm}$ semi-lunar skin incision was made on the infra-umbilical plication (Fig. 1B). A $12 \mathrm{~mm}$ trocar was directly inserted (direct trocar insertion [DTI] technique) $[8,9]$.

Only when intra-abdominal adhesion was suspected, the abdominal cavity was observed after insufflating $\mathrm{CO}_{2}$ (Diagnostic laparoscopy). After the removal of trocar, a home-made port was inserted using Alexis wound retractor (Fig. 1C, D) [10].

Only conventional straight instruments including $0^{\circ}$ endoscope probe of $5 \mathrm{~mm}$ diameter, and others were used during the surgical procedure. The first finger ( $5 \mathrm{~mm}$ trocar) of the home-made port was used as a camera port, and ovarian or infundibulo-pelvic ligament was transected by inserting LigaSure (Covidien, Valleylab, Boulder, CO, USA) to the third finger (12 $\mathrm{mm}$ trocar), third and fifth finger port were used as main instruments' portal [3].

After bladder dissection and complete transection of the uterus using monopolar electric instrument, morcellation was done using laparoscopic knife (Lapknife, Red Medical Korea, Seoul, Korea), and then followed by a $10 \mathrm{~mm}$ laparoscopic tenaculum that was inserted through the vaginal tube to extract the uterus out. Vaginal tube was again inserted to maintain pneumoperitoneum during the vault suture.

Vaginal vault suture was attempted at multi-port TLH with different laparoscopic vault closure techniques (continuous or interrupted suture with straight or curved needle). However, at early cases of SPA-TLH in this study, vaginal repair was done due to inexperience in single-port laparoscopic suture technique (Fig. 1E) (Table 1, cases 1-8). Subsequently, the authors performed continuous suture with straight needle (cases $9,14)$, interrupted suture after vaginal auxiliary surgery with straight needle (cases 10, 16-18), Auto Suture (Endo Stitch, Covidien, CT, USA), favorable in accessing deep vaginal cuff (Fig. 1F) (cases 11, 13), continuous suture with curved needle (cases 12, 14, 15), vaginal manual ligation (Fig. 2A) (cases $11-13,15)$, vaginal auxiliary procedure to easily identify vagi- 


\title{
Obstetrics \& Gynecology Science
}

\author{
Vol. 56, No. 6, 2013
}
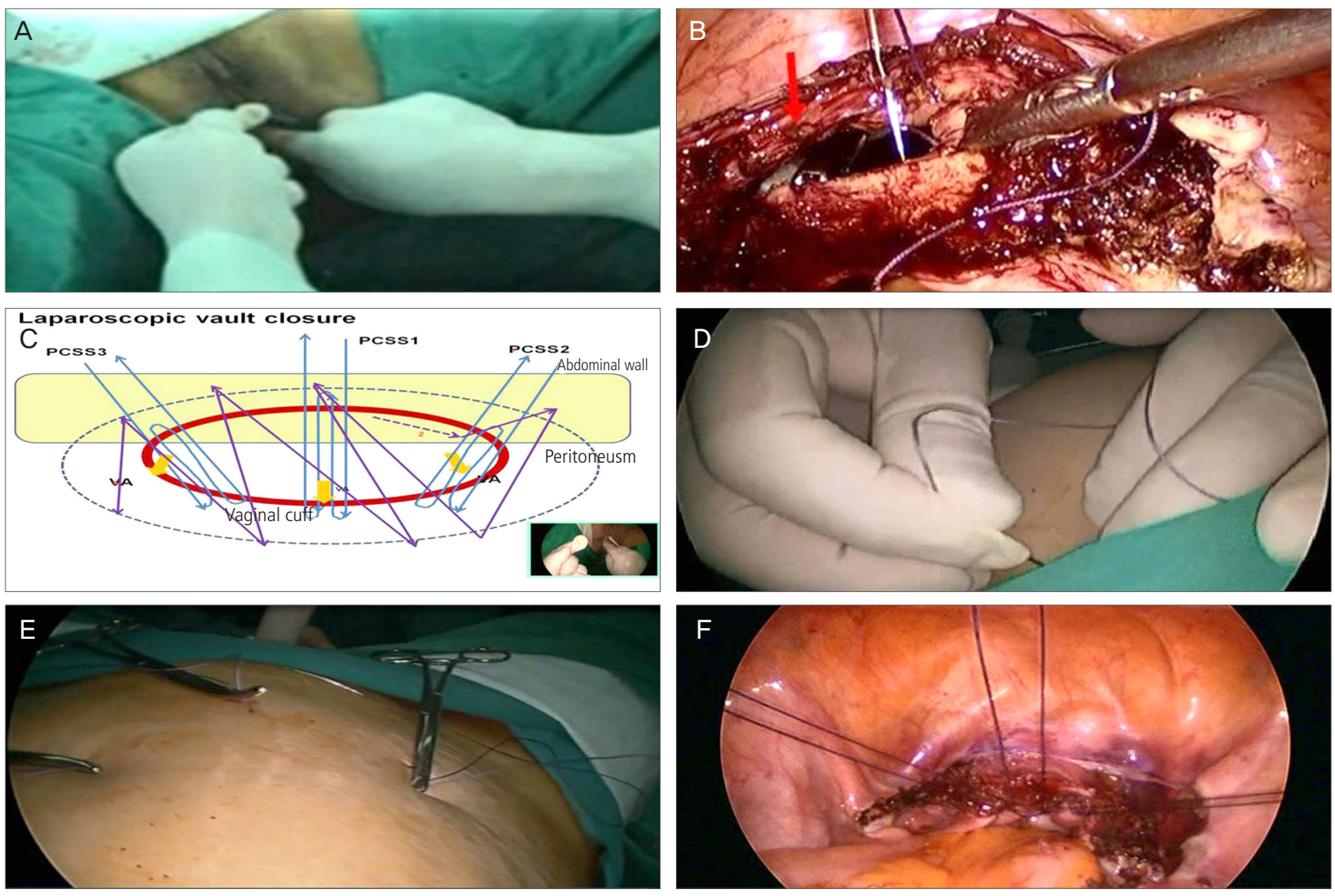

Fig. 2. (A) Vaginal manual ligation. (B) Vaginal assistance by second assistant with traumatic grasper. (C) 3 PCSS with continuous suture in SPA-TLH (schematic view). (D) Insertion of straight needle (1-0 vicryl) to abdomen. (E) Outside view of 3 PCSS. (F) Inside view of 3 PCSS in SPA-TLA. PCSS, percutaneous sling suture; SPA-TLH, single-port access total laparoscopic hysterectomy.

nal cuff (Fig. 2B) (cases 19-21), and others (Table 1). However, the above techniques take long and are difficult, and may generate instability of vault closure. Consequently, percutaneous sling suture (PCSS) (Fig. 2C-F) was performed instead of vaginal auxiliary surgery to easily access vaginal cuff, and then continuous suture was conducted using a curved needle (cases 22-104, 111-119). As experience accumulated, percutaneous sling suture was gradually reduced from three to one, and continuous vault closure without PCSS was performable (cases 105-110).

When suture was done in bilateral angle of vaginal cuff (cases 29-31) instead of PCSS in the center (2 PCSSs), vault closure with continuous suture caused more complication that resulted in the withdrawal of surgery. After experiencing severe bleeding in vaginal cuff (case 65), interrupted suture in an " 8 " shape was made in the bilateral angles of vaginal cuff, and ligation was conducted using a right-angled grasper (cases
64-70). However, the procedure was discontinued due to a rather difficult surgical technique and no further occurrence of vault bleeding.

When the vaginal cuff was barely accessible even after PCSS, Endo Stitch suture was performed (postmenopausal women, cases 61, 81).

Performed ligations were extracorporeal ligation using a knot pusher (Fig. 3A) and intracorporeal ligation using a straight needle holder or right-angled grasper. Vaginal manual ligation was conducted in Endo Stitch suture and continuous vault closure after PCSS (Fig. 2A) (cases 22-119).

\section{Statistical analysis}

Statistical analysis was performed using SAS Enterprise guide ver. 4.1 (SAS institute Inc., Cary, NC, USA). Shapiro-Wilk test was conducted to assess normal distribution of variables. Analysis of variance (ANOVA) or Kruskal-Wallis test was per- 


\section{Obstetrics \& Gynecology Science}

Daehyun Park, et al. Laparoscopic vaginal vault closure in SPA-TLH
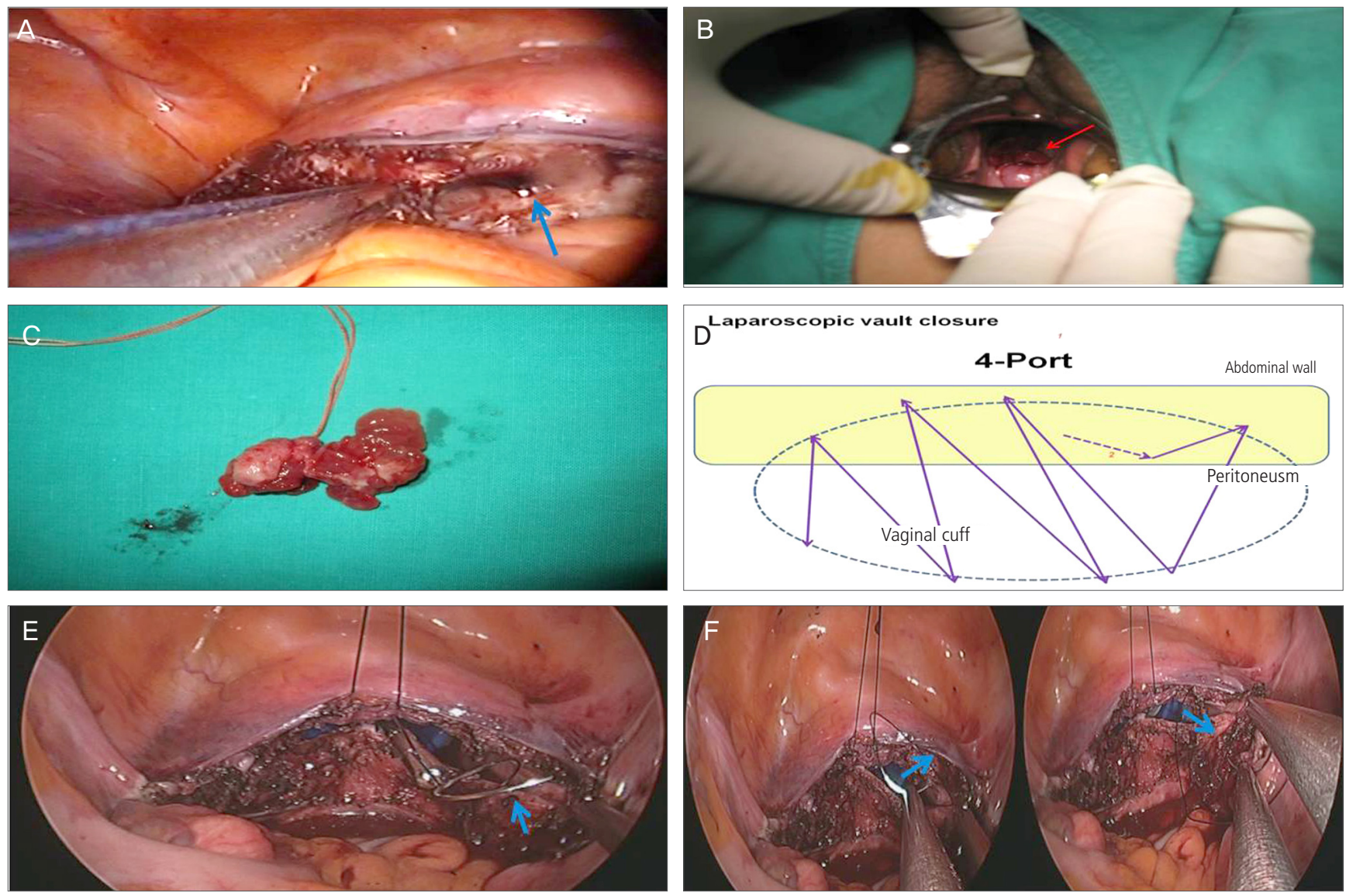

Fig. 3. (A) Extracorporeal tie with knot-pusher (arrow: vaginal assistance). (B) Vaginal vault prolapsed after SPA-TLH (salpinx). (C) Prolapsed salpinx removed after ligation. (D) Vault suture in 4-port TLH (continuous suture, schematic view). (E) Insertion of Monocryl 1-0 for vault closure (arrow: needle). (F) Continuous suture on cut-end of vesicocervical (left arrow), uterosacral (right arrow) ligament. SPA-TLH, single-port access total laparoscopic hysterectomy.

formed for the comparison of continuous variables according to normality. For post-hoc test, Tukey test was used for ANOVA and bon or sidak or Holm was used for Kruskal-Wallis. Moreover, chi-square test or Fisher's exact test were used for the comparison of nominal variables. $P$-values of less than 0.05 were considered statistically significant.

Endo Stitch suture groups (suture along in 2 cases, suture after PCSS in 2 cases) could not satisfy normality in continuous vault closure without PCSS and vaginal suture groups. This study used Kruskal-Wallis test for the analysis of data as a parametric test. For the parametric test, Wilcoxon rank sum test was performed multiple times for post-hoc test, and the difference was modified using Bonferroni, Sidak, or Holm method.

Median was 44.5 minutes (range, 19-56 minutes) in PCSS group. Median was 22 minutes (range, 16-31 minutes) in a group performed with continuous vault closure without PCSS.
Operation time of no PCSS group was almost same as vaginal suture (20 minutes) (Table 1) in vault closure suture. However, statistically significant difference was not found in all modified $P$-values $(P>0.05)$ (Table 2).

\section{Results}

SPA-TLH was performed to 152 patients during this investigation and single-port laparoscopy was completed in 119 cases (78\%). However, 24 cases (16\%) were converted to multiport laparoscopy (2D), and 9 cases (6\%) were converted to laparotomy.

Among 119 cases, 8 cases (7\%) underwent vaginal suture (median, 20 minutes; range, 15-44 minutes), 111 cases (93\%) underwent laparoscopic suture. In laparoscopic suture group, 4 cases (3\%) received extracorporeal ligation (Fig. 3A) using a 


\section{Obstetrics \& Gynecology Science}

Vol. 56, No. 6, 2013

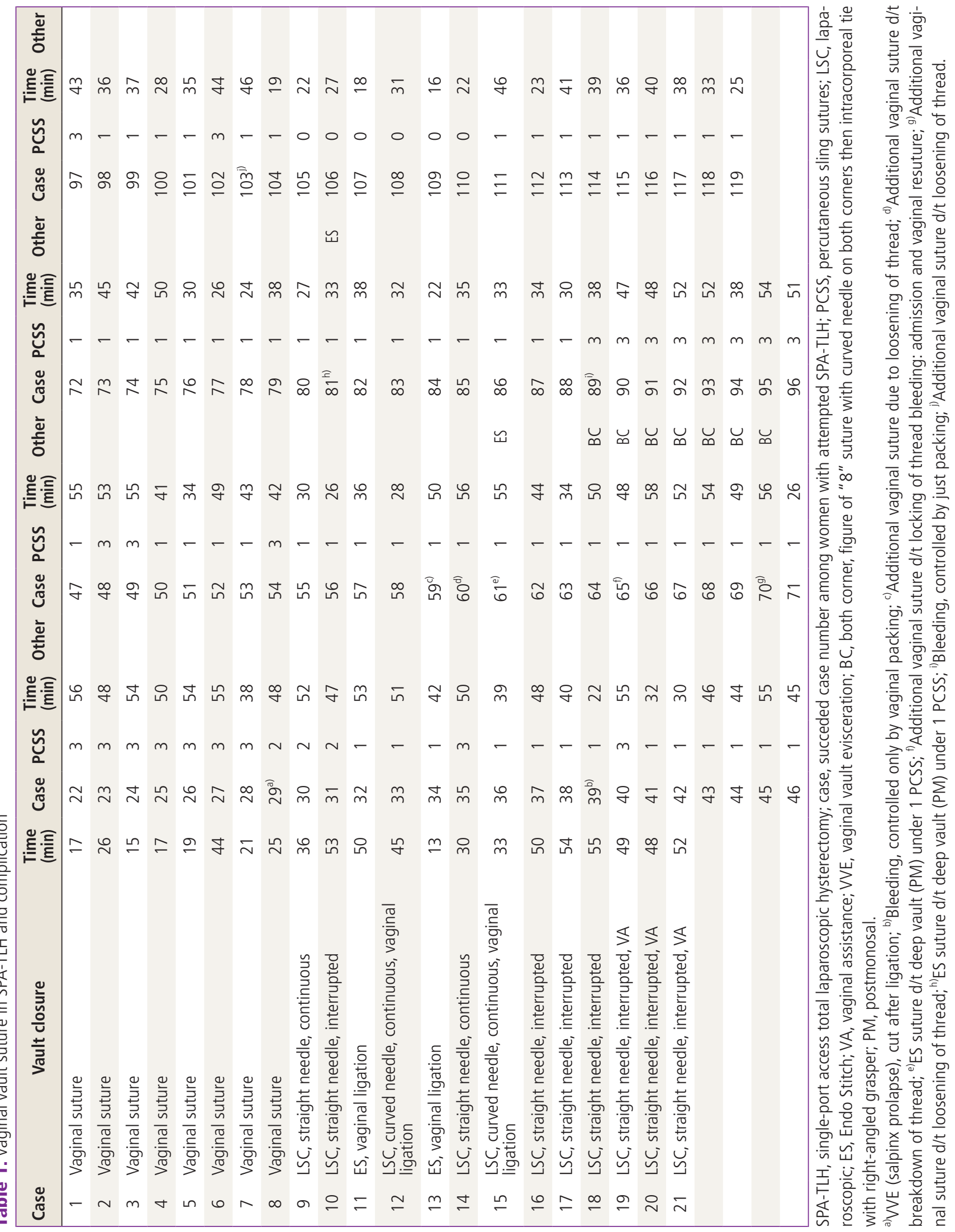




\section{Obstetrics \& Gynecology Science}

Daehyun Park, et al. Laparoscopic vaginal vault closure in SPA-TLH

Table 2. Vaginal vault repair

\begin{tabular}{|lccccc|}
\hline Group & $\mathbf{n}$ & Median & Lowest & Highest & Adjusted $\boldsymbol{P}$-value \\
\hline Vaginal & 8 & 20 & 15 & 44 & - \\
LSC continuous & 4 & 52 & 48 & 55 & - \\
LSC interrupted & 7 & 34.5 & 30 & 45 & - \\
Endo Stitch & 2 & 31.5 & 13 & 50 & - \\
PCSS & 92 & 44.5 & 19 & 56 & $>0.05$ \\
PCSS 0 & 6 & 22 & 16 & 31 & $>0.05$ \\
\hline
\end{tabular}

LSC, laparoscopic; PCSS, percutaneous sling sutures.

knot pusher after additional interrupted suture, since intracorporeal ligation was difficult after continuous suture (median, 34.5 minutes; range, 30-45 minutes) using a straight needle. 7 cases (6\%) underwent extracorporeal ligation using a knot pusher after laparoscopic interrupted suture (median, 52 minutes; range, 48-56 minutes). After the suture using Endo Stitch in 2 cases (2\%; median, 31.5 minutes; range, 13-50 minutes), the suture needle was cut and both ends of the suture materials were taken out from the vagina and manual ligation was performed. One to three PCSS was carried out in 92 cases $(77 \%)$, and then vaginal manual ligation was done after continuous suture (median, 40 minutes; range, 19-56 minutes). Vaginal manual ligation was conducted in 6 cases (5\%) after continuous vault closure without PCSS (median, 22 minutes; range, 16-31 minutes) (Tables 1, 2).

Since the introduction of PCSS in vault closure suture (in case 22nd, 28th attempt), there was no withdrawal of laparoscopic vault suture intraoperatively due to the complexity of the procedure, and no conversion to vaginal suture (Table 1).

Vaginal bleeding occurred in three patients of PCSS group (Table 1, cases 39, 65, 89). Among those, pressure was applied partially to the bleeding site through the vagina in two cases (cases 39,89$)$ to stop bleeding. A case (case 65) was reinforced with vaginal suture after laparoscopic continuous suture in the initial surgery, massive bleeding occurred at the left vault closure angle 4 weeks later the patient was discharged. The bleeding site was sutured in an " 8 " shape vaginally using vicryl 1-0.

A fallopian tube was removed vaginally in a case due to the detection of prolapse during follow-up (case 29) (Fig. 3B, C).

Among cases with laparoscopic suture, vaginal suture was additionally performed after the laparoscopic suture in cases suspected of incomplete suture, and with problems (relaxed, tangled, and broken sutures) during vaginal manual ligation (5 cases) (Table 1, cases 59, 60, 70, 103). Endo Stitch suture was performed in cases (cases 61,81 ) inaccessible to the vaginal cuff despite the conduction of PCSS (Table 1).

\section{Discussion}

Laparoscopic vaginal vault closure is difficult and a timeconsuming procedure in SPA-TLH even though an operator is fully experienced with laparoscopic vault closure in 4-port TLH (Fig. 3D). In the early period of this study, the failure of laparoscopic vault suture led to vaginal suture (cases 1-8) (Fig. $1 \mathrm{E})$.

Laparoscopic suture is classified into suture and ligation. Two barriers must be resolved in order to complete laparoscopic vault closure successfully. First, vaginal cuff needs to be firmly grasped in order for the suture needle to penetrate vaginal cuff for successful suture. Although continuous suture using a straight needle (case 9), interrupted suture using straight needle (case 10), and continuous suture using curved needle (case 12) were attempted, the grasp of the vaginal cuff to be sutured and penetration of the suture materials through vaginal cuff were difficult. Endo Stitch suture (cases 11, 13) (Fig. 1F) was convenient and effective in accessing and suturing deep seated vaginal cuff, but was not suitable as a primary suture technique. Reasons are as follows: 1) Needle often falls out during suturing process because the thickness of autosuture needle is short and vaginal cuff is thick. 2) The rupture of the vault closure is possible despite the use of the thickest suture materials (1-0) during continuous suture. 3) Although Endo Stitch is very convenient once an surgeon is skilled with its specialized ligation technique, applying its ligation method in single-port surgery is considerably difficult. 4) Endo Stitch suture is expensive because the needle holder is disposable. Continuous suture using a straight or curved needle (cases 14, 15), and interrupted suture using straight 


\section{Obstetrics \& Gynecology Science}

Vol. 56, No. 6, 2013
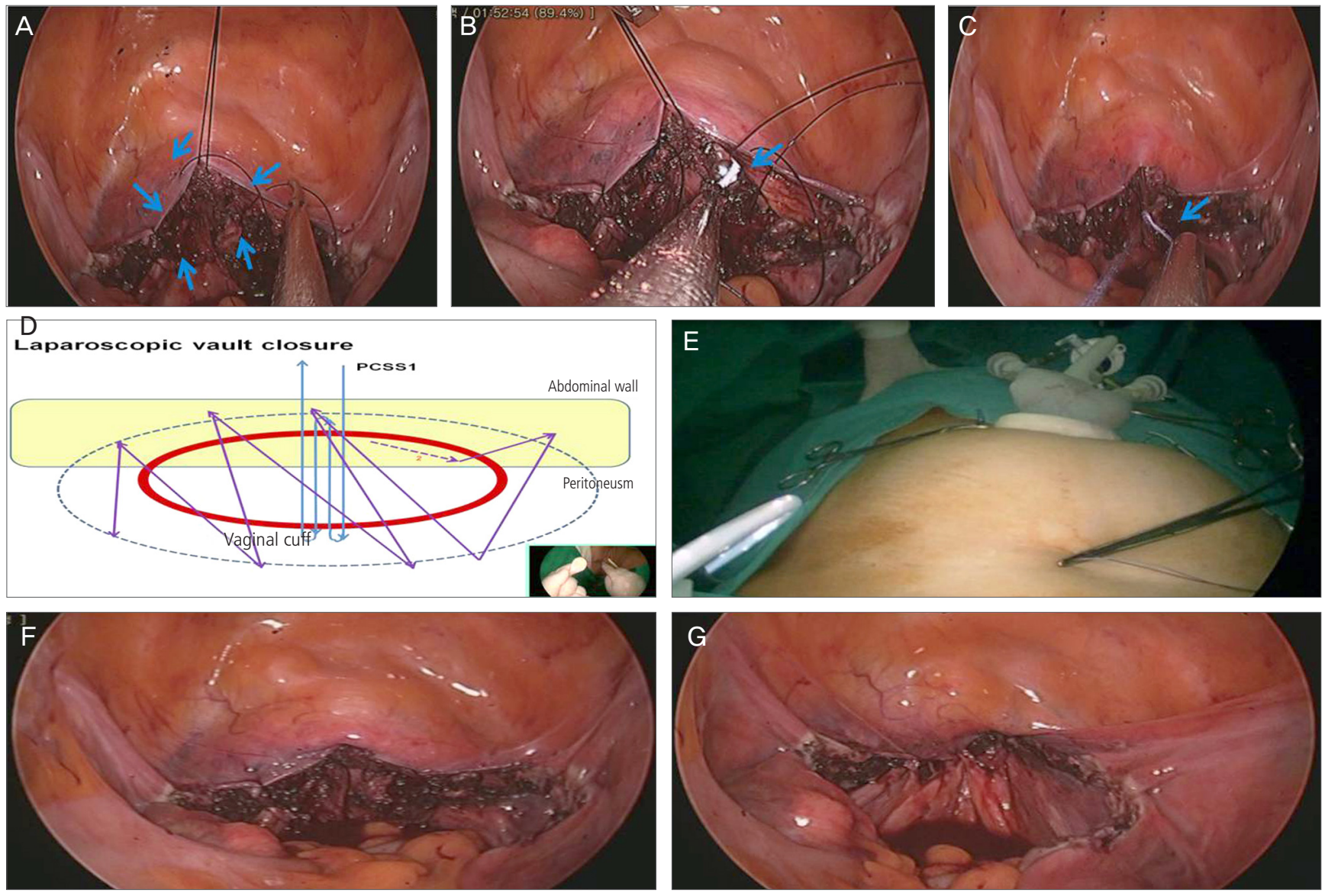

Fig. 4. (A) Continuous suture of pelvic peritoneum. (B) Retrieval of Monocryl. (C) Extracorporeal knot tying with knot-pusher. (D) 1 PCSS with continuous suture in SPA-TLH (schematic view). (E) Outside view of 1 PCSS. (F, G) View of vaginal vault before and after vaginal ligation. PCSS, percutaneous sling suture; SPA-TLH, single-port access total laparoscopic hysterectomy.

needle (cases 16-18) were performed again, since vaginal cuff to be pierced with suture needle is difficult to access. For this reason, we performed vaginal auxiliary procedure (cases 19-21) that retracts vaginal cuff to be sutured by inserting a laparoscopic traumatic forceps to the pelvis through the vaginal tube by second assistant (Figs. 2B, 3A).

However, vaginal auxiliary procedure was uneasy and led to unsatisfied results. To resolve the problem, PCSS (Fig. 2CF) was performed and vaginal cuff was pulled to the anterior and superior direction.

The center of the vaginal cuffs (anterior and posterior) were sutured with a straight needle which was inserted intraabdominally through the abdominal wall, and after pulling out the needle outside through the abdominal wall, both ends of the suture were fixed together with the Kelly's clamp (Fig. $2 \mathrm{C}-\mathrm{F})$.

The difficulty of continuous suture in vaginal cuff was greatly reduced after the retraction to the upper part with three percutaneous sling sutures. Monocryl suture material was inserted intra-abdominally (through vaginal tube or umbilical port) (Fig. 3E) and passed through secured end of vesicocervical ligament of uterus in-to-out at the left corner. Then passing through the secured end of uterosacral ligament out-to-in (Fig. 3F), suture materials passed through slightly left part of the initial suture site of vaginal cuff and then retracted. After performing continuous suture in the rest of the vaginal cuff, suture materials passed through both secured end of right vesicocervical ligaments of uterus and uterosacral ligament in turn and then retracted. Continuous suture was made from the right pelvic peritoneum to the left pelvic peritoneum (Fig. $4 \mathrm{~A})$, and then the suture needle was taken out through the vagina using a grasper inserted intra-abdominally through vaginal tube (Fig. 4B). After cutting the needle of PCSS extracorporeally, suture materials were retracted intra-abdominally using a grasper, and then eliminated extracorporeally through the navel trocar. Subsequently, extracorporeal ligation was 


\section{Obstetrics \& Gynecology Science}

Daehyun Park, et al. Laparoscopic vaginal vault closure in SPA-TLH
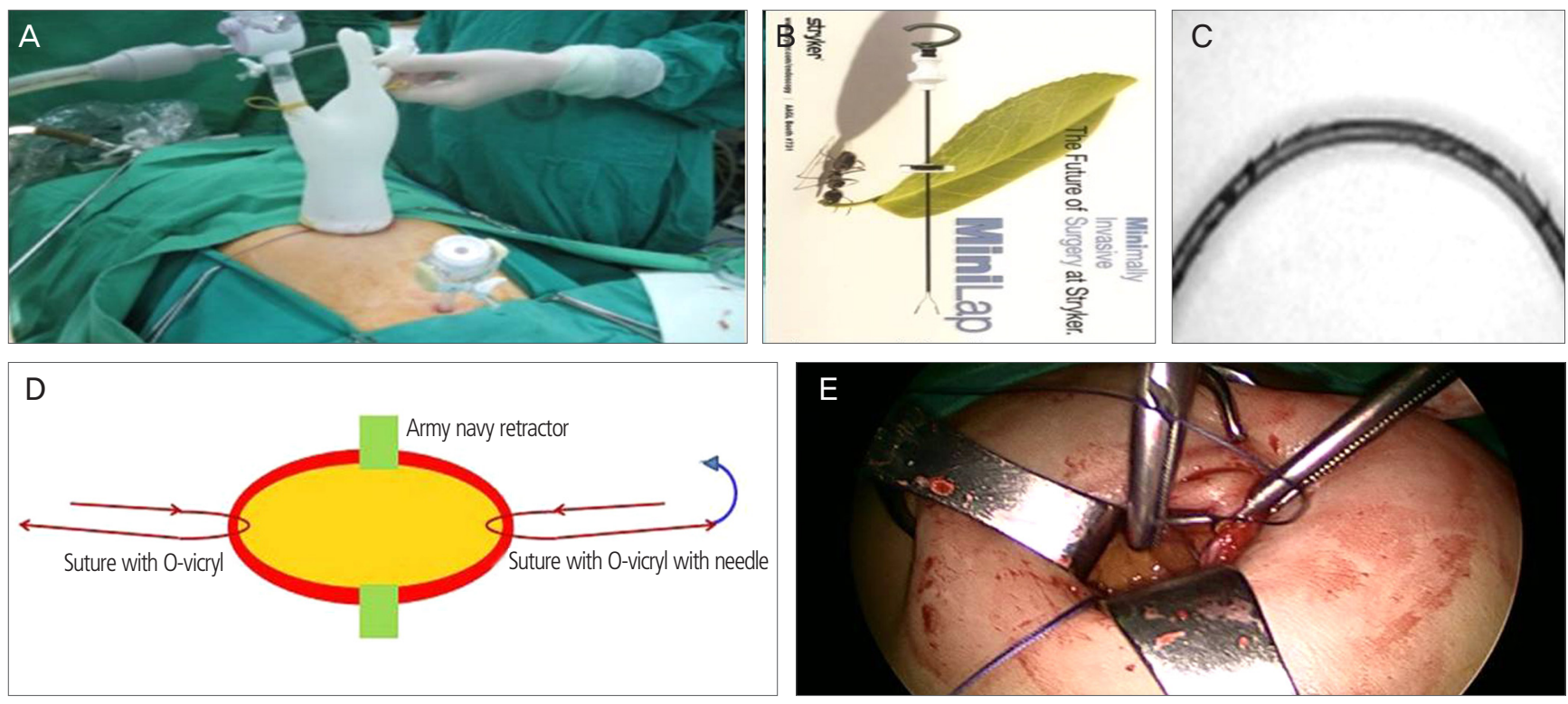

Fig. 5. (A) Outside view of 2-port laparoscopy. (B) MiniLap. (C) Barbed suture. (D) Anchoring sutures for easier port-site closure sckematic view. (E) Anchoring sutures for easier port-site closure.

done using knot pusher and cut (Fig. 4C), followed by a tight vaginal manual ligation with monocryl suture materials (Fig. 2A).

After experienced with PCSS with continuous suture (Fig. 2C) using three PCSSs to some degree, because 3 PCSSs requires considerable amount of time, and PCSSs of vaginal cuff and continuous suture materials sometimes could be tangled during continuous suture, continuous suture was attempted after performing PCSS with two sutures at both angles of vaginal cuff (Table 1, cases 30-32). However, this method was discontinued since continuous suture was more complex due to the absence of suture in the center. Continuous suture was performed by conducting a single PCSS in the center (Fig. $4 D, E)$. Later, authors were skilled with the surgical technique, continuous vault closure without PCSS was performable (Table 1 , cases 105-110).

This laparoscopic vaginal vault closure technique is anticipated to prevent vaginal vault prolapse in the future. According to the results of the follow-up, vault closure sites were located about $1.5 \mathrm{~cm}$ superiorly higher than those of patients with other surgeons' surgery (Fig. $4 \mathrm{~F}, \mathrm{G}$ ). When problems arose during the vault closure suture, using no or single PCSS suture, the surgical technique was then converted to 1 to 3 PCSSs.

When the second trocar was avoidably inserted in the single-port laparoscopic surgery, the conversion to two-port surgery was avoided by performing PCSS instead (Fig. 5A). In this case, PCSS is often called SPA-Rescue. Although the surgical instrument called MiniLap (Fig. 5B) is introduced, the cost of this device is very expensive. To prevent the relaxation of suture materials already sutured during continuous suture, special suture materials (Barbed suture) (Fig. 5C) is introduced. However, these expensive suture materials are unnecessary when vault closure is implemented like in this study.

The second barrier is ligation after suture. After finishing laparoscopic vault suture, extracorporeal ligation using a knot pusher was relatively easier (Fig. 3A). However, extracorporeal ligation after continuous suture requires another interrupted suture, and intracorporeal ligation is very difficult technique. After completing vault closure suture, vaginal manual ligation was done by taking out suture materials through vaginal tube (Fig. 2A).

The suture performed in this study is characterized by maintaining loose, not continuous tight retraction after suture to prevent the relaxation of suture materials which is the greatest weakness of continuous suture. However, retraction states was maintained slightly less tight and tightened during vaginal ligation in this study. For continuous retraction throughout the suture, the role of additionally inserted forceps is unnecessary. Vaginal manual ligation is the easiest and fastest technique that does not require period to be skillful.

In single-port laparoscopic surgery, Hässon technique with manual incision is commonly used by most operators home and abroad in open wound formation after making perpen- 


\section{Obstetrics \& Gynecology Science}

Vol. 56, No. 6, 2013

dicular incision penetrating the navel (Fig. 1B). In this study, semi-lunar transverse incision was made along with infraumbilical plication, and then DTI technique was used instead of veress needle technique in which trocar is inserted after pneumoperitoneum formation with Veress needle. The size of incision was no larger than $15 \mathrm{~mm}$.

$\mathrm{CO}_{2}$ gas was insufflated only in case suspected with intraabdominal adhesions due to the history of laparotomy and others, and the patients' state was observed laparoscopically (diagnostic laparoscopy). When further diagnostic laparoscopic exploration was unnecessary, trocar was removed. Anchoring suture (Fig. 5D, E) was performed in both sides of the open wound, and then a home- made port was inserted using Alexis wound retractor (Fig. 1C, D).

After making a semi-lunar incision in infra-umbilical plication, direct insertion of trocar passing through the navel shortens the time for open wound formation and suturing compared with modified Hässon technique. In addition, gas leakage through the outer wall of trocar in umbilical port was an insignificant problem during multi-port conversion. The authors were able to avoid unconsciously enlarging open wound during vertical incision.

The average time taken for direct trocar insertion after making semi-lunar incision in infra-umbilical plication was 214 seconds (145-300 seconds) in 18 cases of the study. In contrast, the average time taken for making vertical incision inside umbilicus was 277 seconds (160-485 seconds) with Hässon technique performed by other surgeons in 18 cases [3].

In this study, additional vaginal suture was performed due to relaxed, tangled, or broken suture materials after laparoscopic vault closure suture (5 cases, cases 59, 60, 65, 70, 103), and Endo Stitch suture was made after PCSS due to too deep vault closure (2 cases, cases 61,81$)$. Although vaginal vault evisceration such as bowel and omentum due to the rupture of vault closure were not developed [11,12], vault bleeding occurred in 3 cases. We experienced a case of prolapse of the uterine tube (case 29) (Fig. 3B, C).

Among 3 cases of vaginal bleeding (cases 39, 65, 89), only pressure was applied to the bleeding site of vault closure to stop the bleeding in 2 cases. In the other case, bleeding was stopped by performing an " 8 " shape suture vaginally with vicryl $1 / 0$. In a case with re-suture, the patient was modified with vaginal suture after laparoscopic continuous suture. However, massive bleeding occurred 4 weeks later after the patient was discharged. The bleeding site was sutured with " 8 " shape vaginally, and then blood transfusions was conducted (case 65). Afterward, "8" shape interrupted suture was performed bilaterally after PCSS in the center. Although intracorporeal ligation was done using right-angled grasper (cases 64-70), intracorporeal ligation was not performed anymore because there were no longer severe vaginal bleeding and this method required considerable time.

Usually surgeons fully experienced with TLH using 4 ports attempted SPA-TLH. However, performing laparoscopic vaginal vault suture in single-port total hysterectomy is technically very complicated and leads to withdrawal in the middle of the surgical procedure. Consequently, the surgical technique is converted to vaginal suture in most cases. Laparoscopic continuous vault closure and vaginal manual ligation using PCSS meet the conditions of new TLH introduced by Reich.

Laparoscopic vault closure using PCSS in SPA-TLH only with cost-effective conventional straight instruments is the best way to overcome the barrier by considerably reducing surgical withdrawal rate of laparoscopic vault closure and shortening the learning curve to date.

\section{Conflict of interest}

No potential conflict of interest relevant to this article was reported.

\section{References}

1. Reich H, DiCaprio J, McGlynn F. Laparoscopic hysterectomy. J Gynecol Surg 1989;5:213-6.

2. Langebrekke A, Qvigstad E. Total laparoscopic hysterectomy with single-port access without vaginal surgery. J Minim Invasive Gynecol 2009;16:609-11.

3. Park YS. Current trends of gynecologic surgery in the 21st century: scarless surgery. Korean J Gynecol Endosc Minim Invasive Surg 2010;22:69-89.

4. Canes D, Desai MM, Aron M, Haber GP, Goel RK, Stein RJ, et al. Transumbilical single-port surgery: evolution and current status. Eur Urol 2008;54:1020-9.

5. Jung YW, Kim SW, Kim YT. Recent advances of robotic surgery and single port laparoscopy in gynecologic oncology. J Gynecol Oncol 2009;20:137-44.

6. Pelosi MA, Pelosi MA 3rd. Laparoscopic hysterectomy 


\section{Obstetrics \& Gynecology Science}

Daehyun Park, et al. Laparoscopic vaginal vault closure in SPA-TLH

with bilateral salpingo-oophorectomy using a single umbilical puncture. N J Med 1991;88:721-6.

7. Fader AN, Escobar PF. Laparoendoscopic single-site surgery (LESS) in gynecologic oncology: technique and initial report. Gynecol Oncol 2009;114:157-61.

8. Theodoropoulou K, Lethaby DR, Bradpiece HA, Lo $T L$, Parihar A. Direct trocar insertion technique: an alternative for creation of pneumoperitoneum. JSLS 2008;12:156-8.

9. Ahmad G, Duffy JM, Phillips K, Watson A. Laparoscopic entry techniques. Cochrane Database Syst Rev
2008;(2):CD006583.

10. Tai HC, Lin CD, Wu CC, Tsai YC, Yang SS. Homemade transumbilical port: an alternative access for laparoendoscopic single-site surgery (LESS). Surg Endosc 2010;24:705-8.

11. Park YS. Total laparoscopic hysterectomy: self-study. Seoul: Gabon Med Co.; 2008.

12. Park $D$, Jeong $H$. Vaginal vault evisceration after total laparoscopic hysterectomy. Korean J Gynecol Endosc Minim Invasive Surg 2012;24:120-6. 